\title{
Development of Attribute Classification Method for Pedestrians Using Plantar Pressure Value
}

\author{
Takuya Tajima, ${ }^{*}$ Takehiko Abe, ${ }^{1}$ and Haruhiko Kimura ${ }^{2}$ \\ Department of System Management, Fukuoka Institute of Technology, \\ 3-30-1 Wajiro-higashi, Higashi-ku, Fukuoka 811-0295, Japan \\ 'Department of Economics, Aichi University, 1-1 Machihata-cho, Toyohashi, Aichi 441-8522, Japan \\ ${ }^{2}$ Graduate School of Natural Science and Technology, Kanazawa University, \\ Kakuma-machi, Kanazawa, Ishikawa 920-1192, Japan
}

(Received October 30, 2015; accepted February 3, 2016)

Keywords: attribute classification, pressure sensor, plantar, pedestrian, SVM

In this study, we aim to develop and improve an attribute classification method for pedestrians using a plantar pressure value. At present, many retail businesses use various methods (e.g., member's card, direct mail, questionnaire, manual classification by staff members) of collecting customer information. However, these methods have some problems. One of the problems is the instability of collecting customer information. The member's card cannot cover all customers. Moreover, manual classification includes dispersion by individual difference. However, using pressure sensors has advantages. One of the advantages is that the pressure sensor does not violate the targeted person's privacy, because the pressure values from the sensors cannot identify one individual from a large indefinite number. Another advantage is that the pressure sensor can be measured without being affected by the environment (e.g., lighting, field of view, focus range). Our method of attribute classification uses four feature quantities, which are the $\mathrm{CoG}$ (center of gravity in the $X$-axis and the $Y$-axis), plantar area, and total pressure value. In the experimental results, the classification accuracy for gender was $72.19 \%$ and that for three age groups was $42.33 \%$.

\section{Introduction}

According to a white paper on the aging society of Japan published in 2013, 24.1\% of the Japanese population consists of people who are over 65 years old. Moreover, the percentage of aged people is increasing. The Cabinet Office in Japan predicts that the percentages of aged people will be $33.4 \%$ in 2035 and $39.9 \%$ in 2060 . $^{(1)}$ Therefore, about $40 \%$ of the Japanese population will be over 65 years old in the near future. This percentage of aged people is the highest level in the world. This could cause a huge and unprecedented social problem. On the other hand, in Japan, there are many aged people who have considerable leisure time and much money (the percentage of such aged people is about $70 \%$ ). Japanese companies call such people "active seniors". Thus, many companies propose various products and services for these active seniors. Retail businesses, such as supermarkets, regard the active seniors as the most important marketing target because these active seniors increase the average sales per customer.

"Corresponding author: e-mail: t-tajima@fit.ac.jp 
Marketing activities in retail businesses are not only focused on mass marketing. For example, area marketing is a marketing approach based on regional characteristics, and target marketing is a marketing approach based on a person's attributes. Those marketing approaches focus on the analysis of attributes and characteristics. ${ }^{(2)}$ Moreover, there are many other approaches, for example, the analysis of point-of-sale (POS) data for finding hot sellers and the analysis of customers' attributes for understanding buying behavior. ${ }^{(3,4)}$ In particular, marketing research data tends to become large. Therefore, many companies adopt data mining and big data processing. Thereby the companies strengthen their ability to plan and promote sales. ${ }^{(5)}$ However, high-quality data is needed for an accurate analysis. If the obtained data includes the problem of strictness, the result can be false. In Japanese convenience stores and fast food shops, the salesclerks add customers' information regarding gender and age group to the marketing research data using his/ her subjective view. In other words, salesclerks look at the customers and classify their gender and age group. However, a person's subjective view obviously includes inaccuracy and dispersion. For this reason, that classification method is ineffective, and no one can guarantee the accuracy of the data. This is the one of biggest problems. Moreover, this classification work may be mentally tiring for salesclerks because it requires rapid decision-making. Furthermore, the method of using a salesclerk's subjective view and a POS system can not obtain non-buying customers' data. If you execute the marketing to increase sales value, you should find potential customers and prospects, such as people who come to a shop and do not buy. The system in this study that can acknowledge both buying and non-buying customers is effective for marketing.

In addition, there is some research on biometrics that aims to estimate the attributes of the targeted person, such as gender and age. One of the studies uses facial images for estimating a person's age. In Japan, this method is used for preventing minors from buying cigarettes from cigarette vending machines. Moreover, the method is used for counting by age in marketing activities. However, using facial images involves an invasion of privacy. Additionally, this method has the probability of misestimating a person's age. Furthermore, despite taking face images from videos or cameras, there are people who feel discomfort and stress even when they are being watched by security cameras. Naturally, it is not desirable to use cameras or videos for such people. A system for humans must not cause any negative feeling.

In this study, we aim to develop an attribute classification method for pedestrians using the plantar pressure value. This system is used for retail businesses such as convenience stores or supermarkets, and it does not give any stress and discomfort. Furthermore, this system will relieve salesclerks from the mental work of momentarily classifying a customer's gender and age group. Consequently, this system can contribute to the improvement of the work environment.

There is a similar study that uses pressure sensors. ${ }^{(6)}$ The most important difference between that study and this study is the number of types of classifications. In this study, the classification classes are age group and gender, whereas in Ref. 6, only age group classification is carried out. Moreover, the methods and the type of sensors that were used to classify are different. In addition, we proposed similar methods in our studies. ${ }^{(7-9)}$ The differences between our previous studies and this study are feature values. In our previous studies, we used combinations of feature values that resulted in high accuracy in preliminary experiments. However, there is a probability that the accuracy will be lower depending on data combination. Therefore, we selected 4 feature values for contribution to high classification accuracy in this study. As mentioned, this system uses pressure sensors. In the next section, we explain the sensors and the method. 


\section{Sensor and Method}

\subsection{Pressure sensor}

First, we show the specifications of the pressure sensor in Table 1 and the details of the pressure sensor in Fig. 1.

This pressure sensor is made by Xiroku Inc., ${ }^{(10)}$ and it is called an "LL sensor". ${ }^{(11)}$ The shape of this pressure sensor is similar to that of a panel or a small carpet. One reason for using this sensor is that there is no individual classification method from an unspecified large number of people. At present, individual classification methods using pressure values or pressure distributions have not been established. Thus, pressure values cannot be used for individual identification. Thereby, this system does not invade privacy. The second reason is robustness to observe environments. The pressure sensor can be used without lighting and can also work while being exposed to temperature, humidity, noise, physical obstacles, and silhouettes of people or objects. The third reason is that this system can be used in parallel with other attribute classification systems that use facial images from cameras or videos. Thereby, when this system and other systems work together, it is expected to create a classification system with the highest level of accuracy that has ever been seen.

In this experiment, three pressure sensors worked as one panel virtually by using measurement software. Figure 2 shows the installation of the measurement system with 3 pressure sensors. An examinee walks straight from the left side to the right side with his/her normal speed and step. Figure 3 shows plantar pressure values by continuous silhouettes.

Table 1

Specifications of pressure sensor.

\begin{tabular}{lc}
\hline Sensor type & Electromagnetic induction \\
\hline Size & $600 \times 600 \mathrm{~mm}^{2}$ \\
Thickness & $2 \mathrm{~mm}$ \\
Sensing point & $3600(60 \times 60) /$ sheet \\
Element separation & $10 \mathrm{~mm}$ \\
\hline
\end{tabular}

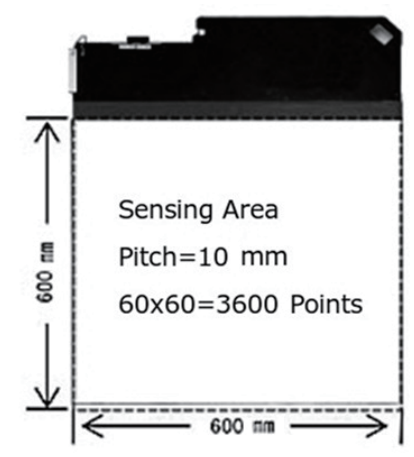

(a)

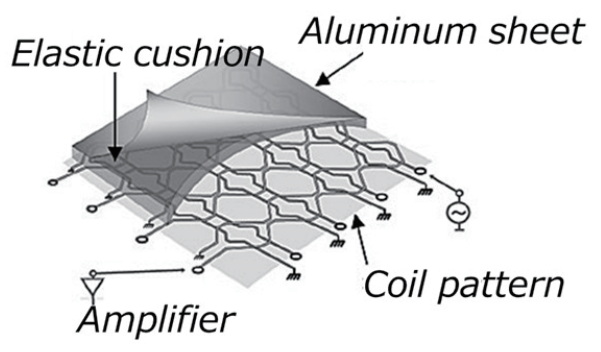

(b)

Fig. 1. Details of pressure sensors. (a) Appearance of sensors and (b) structure of sensor part. 


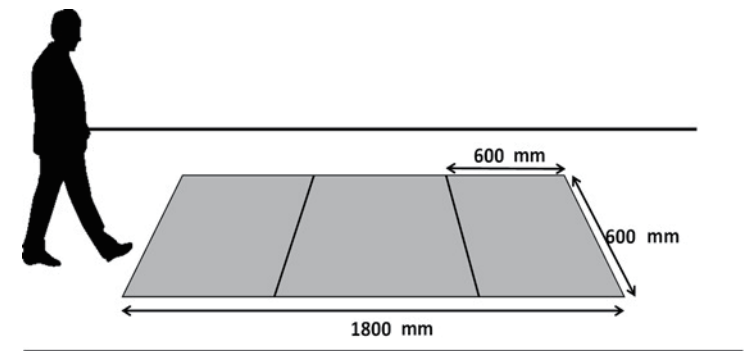

Fig. 2. Installation of measurement system.

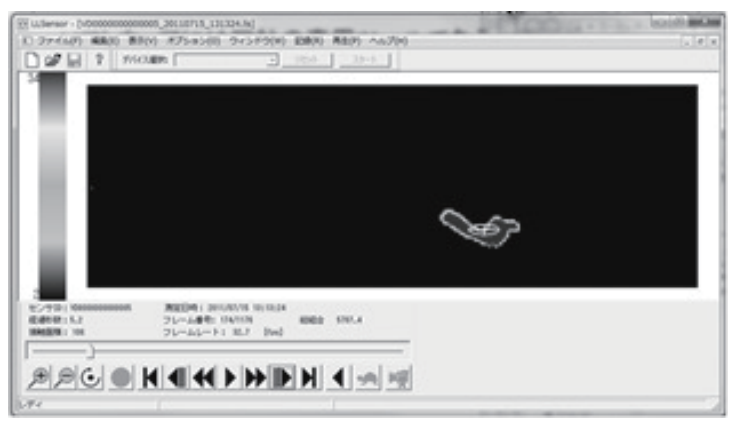

Fig. 3. Measurement software.

\subsection{Walk action measurement system}

As mentioned, the measurement system has 3 pressure sensors. The length is $1800 \mathrm{~mm}$ and its width is $600 \mathrm{~mm}$ (Fig. 2). This pressure sensor detects the pressure value from each cell. Here, the

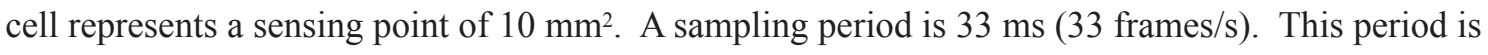
equal to a conventional video camera's sampling period.

The number of examinees was 153 (male, 57; female, 96), and the age range was 20-89 years old. Figure 4 shows the percentage distribution of the examinees by age and gender in this experiment. Each examinee could walk without support. The examinees were shoeless or wore socks. This condition was set by removing the effect of a shoe's shape and sole patterns for the basic experiment. We did not give a specific instruction on how examinees should walk. We only said to all the examinees: "Please walk as usual". Each examinee walked 10 rounds as one experiment. The total number of round was 1530 (153 persons $\times 10$ rounds).

\section{$2.3 \quad$ Feature values}

In this experiment, there is a difference in experimental time because each examinee has an individual walking step and speed. Therefore, all sampling data were normalized as 30 frames of data. One reason for this is that we attempted to decrease data volume and hence processing time. Another reason is that all the examinees could put both feet on the pressure sensor in a walking motion within less than $1 \mathrm{~s}$ (33 frames). Therefore, the first frame was the moment of landing an examinee's heel on a pressure sensor at the start of obtaining data. In addition, by preliminary experiment, data obtained under $20 \mathrm{~g}$ of pressure was considered noise. Thus, we removed such data. Thereby, one data point has feature values of 30 frames in the time series. The feature values in this study were the center of gravity in the $X$-axis, the center of gravity in the $Y$-axis, total pressure value, and plantar area. The coordinates of sensing points are shown by the $X$-axis (length of pressure sensors) and the $Y$-axis (width of pressure sensor) as $(X, Y)$. For example, from Table 1 and Fig. 3, the $X$-axis is $1800 \mathrm{~mm}$, the $Y$-axis is $600 \mathrm{~mm}$, and the element separation is $10 \mathrm{~mm}$. Thereby, the coordinate of the $X$-axis can be taken as $1-180$, and the coordinate of the $Y$-axis can be taken as $1-60$. Therefore, the coordinate of the upper left is $(1,1)$, and the coordinate of lower right is $(180,60)$. 




Fig. 4. Statistics of examinees' age and gender.

In the following, the definitions and equations of the 4 feature values are explained. In Eqs. (1)-(4), $i$ is the coordinate on the $X$-axis and has a range of $1-180$, and $j$ is the coordinate of the $Y$-axis and has a range of $1-60$.

(1) Center of Gravity in the $X$-axis: $X_{\mathrm{CoG}}$

$X_{\mathrm{CoG}}$ denotes the walking speed and walking distance to the front. In general, young people can walk faster than elderly people. ${ }^{(12)}$ Therefore, this feature value can contribute to the age group classification. Equation (1) shows the calculation method by $X_{\mathrm{CoG}}$. In Eq. (1), $x_{i}$ is the coordinate of the pressured $X$-axis, $f_{i}$ is the pressure value of the $X$-axis, and $f_{i j}$ is the summed pressure value in the $Y$-axis direction. Moreover, each set of data includes 30 frames. In addition, the meaning of Eq. (1) and later of Eq. (2) is same as the physical center of gravity.

$$
X_{\mathrm{CoG}}=\frac{x_{i} \sum_{j=1}^{60} f_{j}}{\sum_{i=1}^{180} \sum_{j=1}^{60} f_{i j}}
$$

(2) Center of Gravity in the $Y$-axis: $Y_{\mathrm{CoG}}$

$Y_{\mathrm{COG}}$ denotes the sideways swing distance. The sideways swing distance approximates the step width. Figure 5 shows the relationship between step width and $Y_{\mathrm{CoG}}$. The step width has a positive correlation with age. Namely, elderly people have a lager step width than young people. Moreover, elderly males have a larger step width than elderly females. ${ }^{(12)}$ Therefore, this feature value can contribute to both age group and gender classifications. Equation (2) is the calculation method of $Y_{\mathrm{CoG}}$. In Eq. (2), $y_{i}$ is the coordinate of the pressured $Y$-axis, $f_{i}$ is the pressure value of the $Y$-axis, and $f_{i j}$ is the summed pressure value in the $X$-axis direction.

$$
Y_{\mathrm{CoG}}=\frac{y_{j} \sum_{i=1}^{60} f_{i}}{\sum_{j=1}^{60} \sum_{i=1}^{180} f_{i j}}
$$

(3) Total pressure value: $w$

The total pressure value that is shown is the summed pressure value per frame. Equation (3) is the calculation method for the total pressure value. In general, a male is heavier than a female. 


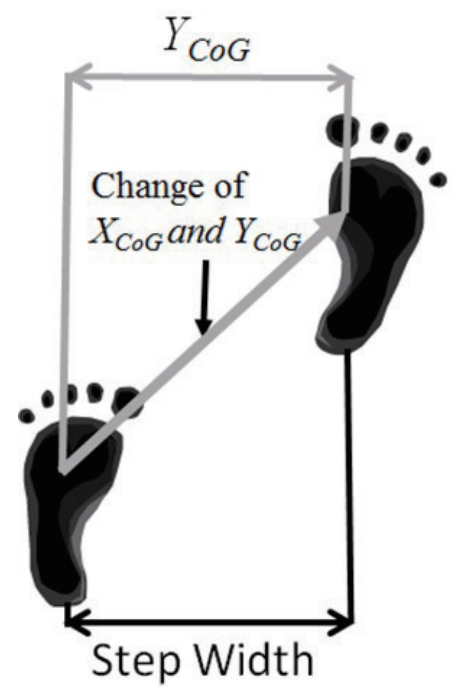

Fig. 5. Image of relationship between step width and $Y_{\mathrm{CoG}}$.

Therefore, this feature value can contribute to gender classification. Moreover, elderly people are apt to limp while walking. In contrast, young people can walk with powerful steps. Therefore, this feature value can also contribute to age classification. In addition, this value is not simple human weight as it means load per frame.

$$
w=\sum_{i=1}^{180} \sum_{j=1}^{60} f_{i j}
$$

(4) Plantar area: $s$

The plantar area is the summed number of pressured sensing points. Equation (4) is the calculation method for the plantar area. In general, a male's plantar area (e.g., shoe size) is larger than a female's plantar area. Therefore, this feature value can contribute to gender classification. In Eq. (4), $a_{i j}$ is the condition of the sensing point. A pressured sensing point shows 1, and a nonpressured sensing point shows 0 . In addition, sensing points are set one per $1 \mathrm{~cm}$. Therefore, a 1 for this value means $1 \mathrm{~cm}^{2}$.

$$
s=\sum_{i=1}^{180} \sum_{j=1}^{60} a_{i j}
$$

\subsection{Classification data}

In this section, the creation of classification data is explained. Table 2 shows the data format of feature values. In Table $2, x_{1}-x_{30}$ are $X_{\mathrm{COG}}, y_{1}-y_{30}$ are $Y_{\mathrm{CoG}}, w_{1}-w_{30}$ are the total pressure values, and $s_{1}-S_{30}$ are the plantar areas for 30 frames. In addition, "IS" means "instruction signal". There are 
Table 2

Data format and example of data.

\begin{tabular}{|c|c|c|c|c|c|c|c|c|c|c|c|c|}
\hline$x_{1}$ & $\ldots$ & $x_{30}$ & $y_{1}$ & $\ldots$ & $y_{30}$ & $w_{1}$ & $\ldots$ & $w_{30}$ & $s_{1}$ & $\ldots$ & $S_{30}$ & IS \\
\hline 55.81 & $\ldots$ & 67.12 & 27.05 & $\ldots$ & 20.80 & 8368 & $\ldots$ & 5938 & 167 & $\ldots$ & 147 & $20 \mathrm{~s}$ \\
\hline
\end{tabular}

two types of "IS". The first type is "M" and "F", where "M" means male and "F" means female. Another type is "20s", "30s", and "50s". "20s" means people who are in their $20 \mathrm{~s}$, "30s" means people who are in their 30-40s, and "50s" means people who are over 50 years old. Moreover, the number of dimensions of data is 121 ( 4 types of feature values for each 30 and "IS").

In this study, the data set is created in 2 patterns. The first is for gender classification, and the second is for age group classification. The first data set includes data for people in their 20-80s (refer to Table 3). The number of examinees is 114 (male, 57; female, 57). The number of data points is 1140 (both data numbers of males and females are 570), because 1 examinee has 10 rounds experimental data. The gender classification is a binary classification. The second data set includes data of people in their 20-70s (refer to Table 4). The number of people in each class of "20s", "30s", and " $50 \mathrm{~s}$ " is 30 . Thus, there are 3 classification classes. Moreover, the total number of examinees is 90 . Each examinee has 10 rounds experimental data. Therefore, the number of data points in one class is 300 . The total number of data points is 900 . Furthermore, we selected examinees in Table 3 and 4 from all examinees in Fig. 4. There is not a selection criteria for examinees, because we selected examinees at random. In addition, the data were obtained in a laboratory and public hall.

In Fig. 4, the distributions of examinees' gender and age groups are uneven. Namely, the numbers of young males and elderly females are larger than the numbers of elderly males and young females. Thereby, there is a probability that uneven distribution of gender affects age group classification accuracy. Conversely, there is a probability that uneven distribution of age group affects gender classification accuracy. However, all data in Fig. 4 are not used. Table 3 and 4 show the examinees data used. Thus, uneven distribution is moderated.

\subsection{Classification Method}

The classification method is the support vector machine (SVM) that was used. ${ }^{(13-15)}$ SVM uses a margin between the hyper plane and the training samples as an evaluation function. When the SVM is unable to separate the observed data into linear space, it realizes the linear separation by mapping data to a higher dimensional space through nonlinear conversion. The method used to greatly reduce the amount of calculation is called the kernel trick. There are several kernel functions, such as linear, polynomial, sigmoid, and Gaussian. Although the SVM performance is affected by the kernel selection and parameters, an effective method for selecting the kernel has not yet been established. In this study, we used the Gaussian kernel because the number of parameters in the Gaussian kernel is small. The equation of the kernel function is Eq. (5). $K\left(x, x^{\prime}\right)$ is the kernel function, and $x$ and $x^{\prime}$ are input data. $\gamma$ is the inverse of the dimensional number of feature data points $(\gamma>0)$. The Gaussian kernel is one of the most used kernels.

$$
K\left(x, x^{\prime}\right)=\exp \left(-\gamma\left|x-x^{\prime}\right|^{2}\right)
$$


Table 3

Number of examinees in each class for gender classification.

\begin{tabular}{lcc}
\hline Age group & Male & Female \\
\hline $20 \mathrm{~s}$ & 22 & 8 \\
$30 \mathrm{~s}$ & 8 & 8 \\
$40 \mathrm{~s}$ & 5 & 8 \\
$50 \mathrm{~s}$ & 7 & 8 \\
$60 \mathrm{~s}$ & 7 & 9 \\
$70 \mathrm{~s}$ & 7 & 9 \\
$80 \mathrm{~s}$ & 1 & 7 \\
\hline
\end{tabular}

Table 4

Number of examinees in each class for age group classification.

\begin{tabular}{lcc}
\hline Age group & Male & Female \\
\hline $20 \mathrm{~s}$ & 22 & 8 \\
$30 \mathrm{~s}$ & 8 & 7 \\
$40 \mathrm{~s}$ & 5 & 10 \\
$50 \mathrm{~s}$ & 5 & 5 \\
$60 \mathrm{~s}$ & 5 & 5 \\
$70 \mathrm{~s}$ & 5 & 5 \\
\hline
\end{tabular}

If the number of calculations of classification accuracy is 1 , the result may be higher or lower depending on biased data. One should check the average classification accuracy. Thus, we calculated the average classification accuracy by cross-validation. The cross-validation in this study is one-to-others. For example, in an experiment with 100 people in the age group classification, data is separated as 99 people learning data and 1 person test data. By this cross-validation method, learning data does not include test data. Therefore, the classification accuracies mean perfect unknown data classification accuracy. The learned SVM model can classify perfect unknown data. Furthermore, this cross-validation method is recalculated 99 more times with changing test data and learning data. Finally, an average accuracy is calculated.

\section{Experimental Results}

Tables 5 and 6 are the experimental results of gender classification. In the tables, the colored cells show the correct classification number and ratios. The total numbers of males and females are both 570 . The average accuracy is $72.19 \%$.

Tables 7 and 8 are the experimental results of age group classification. In the tables, the colored cells show the correct classification number and ratios. The total number of " $20 \mathrm{~s}$ ", " $30 \mathrm{~s}$ ", and "50s" (actual age ranges are 20s, $30-40 \mathrm{~s}$, and $50-70 \mathrm{~s}$ ) is 90 . The average accuracy is $40.22 \%$. We explain the interpretations of those four tables. For example, in the row "male" in Table 5, cell of male and male means the correct classification number. The cell of male and female means the incorrect classification number. This means the number of males classified as female. The interpretations of the other tables are the same.

Figures 6-9 show the averages of $X_{\mathrm{CoG}}, Y_{\mathrm{CoG}}$, the total pressure value, and plantar area in each class. Namely, the averages of $X_{\mathrm{COG}}, Y_{\mathrm{COG}}$, the total pressure value, and plantar area are calculated per frame. Then, each average value is plotted in the frame order. Here, class means gender and 
Table 5

Result of gender classification (in number).

\begin{tabular}{lcc}
\hline & Male & Female \\
\hline Male & $\mathbf{4 1 3}$ & 160 \\
Female & 157 & $\mathbf{4 1 0}$ \\
\hline
\end{tabular}

Table 7

Result of age group classification (in number).

\begin{tabular}{rrrr}
\hline & $20 \mathrm{~s}$ & $30 \mathrm{~s}$ & $50 \mathrm{~s}$ \\
\hline $20 \mathrm{~s}$ & $\mathbf{1 2 2}$ & 86 & 90 \\
$30 \mathrm{~s}$ & 103 & $\mathbf{1 2 5}$ & 95 \\
$50 \mathrm{~s}$ & 75 & 89 & $\mathbf{1 1 5}$ \\
\hline
\end{tabular}

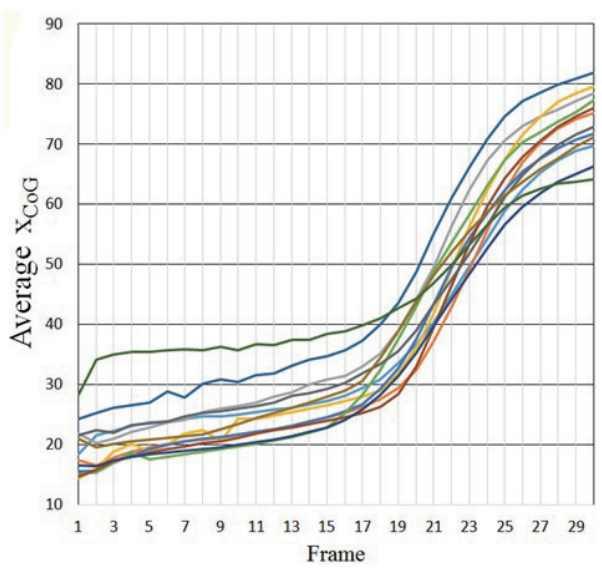

Fig. 6. Average $X_{\mathrm{CoG}}$ in each class.



Fig. 8. Average total pressure value in each class.
Table 6

Result of gender classification (in percentage).

\begin{tabular}{lcc}
\hline & Male & Female \\
\hline Male & $\mathbf{7 2 . 4 6 \%}$ & $28.07 \%$ \\
Female & $27.54 \%$ & $\mathbf{7 1 . 9 3 \%}$ \\
\hline
\end{tabular}

Table 8

Result of age group classification (in percentage).

\begin{tabular}{lccc}
\hline & $20 \mathrm{~s}$ & $30 \mathrm{~s}$ & $50 \mathrm{~s}$ \\
\hline $20 \mathrm{~s}$ & $\mathbf{4 0 . 6 7 \%}$ & $28.67 \%$ & $30.00 \%$ \\
$30 \mathrm{~s}$ & $34.33 \%$ & $\mathbf{4 1 . 6 7 \%}$ & $31.67 \%$ \\
$50 \mathrm{~s}$ & $25.00 \%$ & $29.67 \%$ & $\mathbf{3 8 . 3 3 \%}$ \\
\hline
\end{tabular}

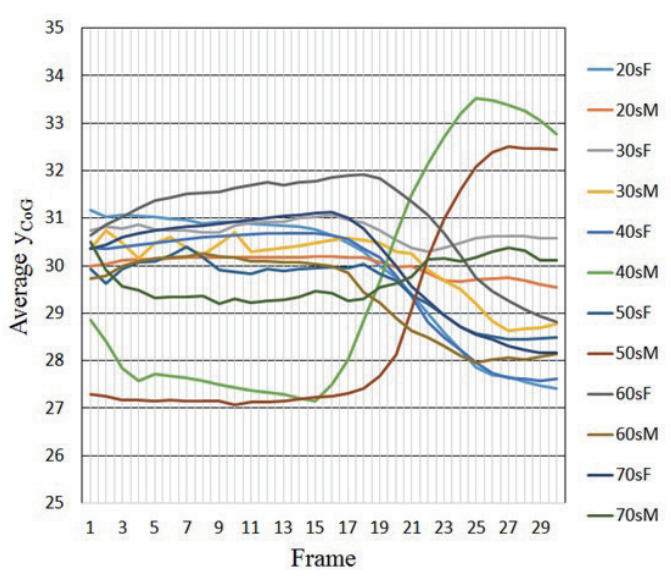

Fig. 7. Average $Y_{\mathrm{CoG}}$ in each class.

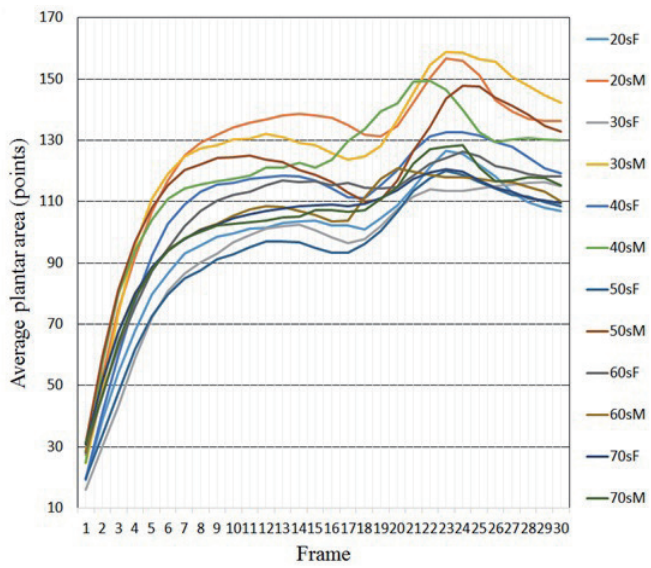

Fig. 9. Average total plantar area in each class.

age group (e.g., 20s male class). In addition, "20sF" along the $Y$-axis on the right of the graph in these figures means $20 \mathrm{~s}$ female class. In these figures, the horizontal axis means frame, and the vertical axis means each average. Each line means each average in each class. In the interpretation 
of Fig. 6, the left side of Fig. 6 means the left side of sensor in Fig. 2. Namely, the left side means the starting place for data collection. The right side means the end of the sensor. The height of Fig. 6 means $X$-axis. Therefore, a point higher on the $X$-axis means farther from left side (length of walking to right side). At 18-25 frame in Fig. 6, the line rapidly rises. This means rapid moving of the center of gravity, and this means going to the front by the next step. Furthermore, Fig. 7 shows swinging to the left and right. In Fig. 7, going up means a swing to the left side, going lower means a swing to the right side. Figures 8 and 9 show average total pressure values and average total plantar areas in each class. Those figures show increases. The reason is that the start of walking is landing of the heel, and then, lading of arch of the feet, and final, landing the toe. Thereby, the total pressure value and the total plantar area increase sequentially in accordance with order of steps.

\section{Discussion}

In Fig. 6, the gradients of lines mean walking speed. Admittedly, the walking speed of a 70s male is slower than those of the other classes. We can understand that aged males walk particularly slow.

In Fig. 7, an increasing $Y_{\mathrm{CoG}}$ means moving to the left side. In contrast, a decreasing $Y_{\mathrm{COG}}$ means moving to the right side. The $Y_{\mathrm{COG}}$ of a 40s male shows a larger width of increasing and decreasing than those of the other classes. Moreover, a 50s male has the second largest width. However, a 20 s male and a 20-30s female do not have a large width. Thereby, we understand that middle aged people walk with a swing.

In Fig. 8, the large vertical value means a strong load. The peak total pressure value at 20-23 frames in 30 s males is the largest. The next largest is that in 40 s males, and then 20 s males. In contrast, the peak total pressure values of 60-70s males are lower than those of young males. Moreover, the total pressure values of many female classes are lower than those of males. Thereby, we understand that aged people walk with a limp.

In Fig. 9, a large value means a large plantar area. Therefore, a large value means a large foot size. In all classes, $30 \mathrm{~s}$ males had the highest peak, then $20 \mathrm{~s}$ males, $40 \mathrm{~s}$ males, and $50 \mathrm{~s}$ males. The lowest is for $30 \mathrm{~s}$ females. Next in the order is $60 \mathrm{~s}$ males, $50 \mathrm{~s}$ females, and $70 \mathrm{~s}$ females. Thereby, we understand that this feature value contributes to gender classification and age group classification in males.

Furthermore, examinees did not wear shoes in these experiments. However, because of actual use, we think that it is necessary to run experiments with shoes. Here, we consider the effect of shoes. The shoes have differences between the genders and ages. For example, children rarely wear formal leather shoes, and males rarely wear high heels. Those differences have a positive effect for classifications of gender and age group. Moreover, people who wear high heels walks warily. Therefore, walking behavior with high heels is different from usual walking. Therefore, we think that wearing shoes can ease classifications.

In Tables 5-8, the average accuracy for gender is $72.19 \%$, and the average accuracy for age group is $40.22 \%$. These accuracies are the same or slightly lower than those in other studies using facial images and facial features. ${ }^{(16-18)}$ However, our classification method can be used with other methods and systems (e.g., the systems using cameras and videos) because our method and system do not use facial images. Therefore, our system can be used in parallel without interrupting other systems. Therefore, there is a strong probability that the classification accuracy can be improved considerably. This point is a strong advantage of our method and system. 


\section{Conclusions}

In this study, we aimed to develop and improve an attribute classification method for pedestrians using the plantar pressure value. Many retail businesses use some methods (e.g., member's card, direct mail, questionnaire, or manual classification by a staff member) for collecting customers' information. However, these methods have some problems. One of the problems is the instability of collecting customers' information. The member's card cannot cover all customers. Moreover, manual classification includes dispersion by individual difference. Using pressure sensors has advantages. One of the advantages is that the pressure sensor does not violate the targeted person's privacy, because the pressure values from the sensors cannot identify an individual from a large indefinite number of people. Another advantage is that the pressure sensor can be measured without being affected by the environment (e.g., lighting, field of view, and focal range).

Our method of attribute classification uses four feature quantities, namely, $X_{\mathrm{CoG}}, Y_{\mathrm{CoG}}$, plantar area, and the total pressure value. In the experimental results, the classification accuracy for gender was $72.19 \%$ and that for three age groups was $42.33 \%$. We propose an improvement in this system. The four feature quantities used in our system were obtained from people without any shoes. Therefore, the system might not be applicable in actual locations such as convenience stores and supermarkets, as many people in these shops wear shoes.

We shall improve our system for future use. Namely, our system will be adapted for people who wear shoes. By doing this, it will be possible to use the system in any organization across the world.

\section{References}

1 Cabinet Office: White Paper on Aging Society (2013) (in Japanese).

2 R. Kobayashi: Regional Studies 38 (2010) 13 (in Japanese).

3 P. M. Guadagni and J. D.C. Little: Marketing Science 2 (1983) 203.

4 S. Gupta: JMR 25 (1998) 342.

5 Y. Hamuro, N. Katoh, Y. Matsuda, and K. Yada: Data Mining and Knowledge Discovery 2 (1998) 391.

6 M. Sudo, Y. Yamashiro, K. Ueno, and H. Kim: JPA 18 (2013) 125 (in Japanese).

7 J. Hasegawa, T. Tajima, T. Abe, and H. Kimura: Transactions of JSPM 18 (2012) 83 (in Japanese).

8 J. Hasegawa, T. Tajima, T. Abe, and H. Kimura: Proc. of the 13th APIEMS Conference (APIEMS, Phuket, 2012) pp. 956-961.

9 J. Hasegawa, T. Tajima, T. Abe, and H. Kimura: Proc. of The 14th APIEMS Conference (APIEMS, Cebu, 2013) (CD-ROM).

10 Xiroku Inc.: Xiroku Inc., http://www.xiroku.com (accessed October 2015).

11 LL sensor: LL sensor, http://www.llsensor.com (accessed October 2015).

12 K. Miyatsuji, J. Sawayama, K. Kawabata, and M. Kaneko: JPA 12 (2007) 165 (in Japanese).

13 K. Tsuda: Institute of Electronics, Information, and Communication Engineers, Transactions 83 (2000) 460 (in Japanese).

14 M. Pontil and A. Verri: IEEE Transactions on Pattern Analysis and Machine Intelligence 20 (1998) 637.

15 V. Vapnik: The Nature of Statistical Learning Theory (Springer-Verlag, New York, 2000).

16 K. Ikuta, H. Kage, K. Tanaka, and K. Kyuma: Technical Report of IEICE 106 (2007) 65 (in Japanese).

17 K. Ikuta, H. Kage, K. Sumi, K. Tanaka, and K. Kyuma: Technical Report of IEICE 107 (2008) 121 (in Japanese).

18 K. Ueki, T. Hayashida, and T. Kobayashi: Technical Report of IEICE 105 (2005) 13 (in Japanese). 